\title{
Determinantes da adesão aos serviços de mobilidade compartilhada: uma investigação empírica no contexto brasileiro
}

Adherence determinants to shared mobility services: an empirical investigation in the Brazilian context

Adelma da Silva Santos[a] $[$, João Carlos Hipolito Bernardes do Nascimento[a] (1), Alexandre Rabêlo Neto[a] (1), Flávia Lorenne Sampaio Barbosa[a] [i]

[a] Universidade Federal do Piauí (UFPI), Floriano, PI, Brasil

Como citar: Santos, A. S., Nascimento, J. C. H. B., Rabêlo Neto, A., \& Barbosa, F. L. S. (2019). Determinantes da adesão aos serviços de mobilidade compartilhada: uma investigação empírica no contexto brasileiro. urbe. Revista Brasileira de Gestão Urbana, 11, e20190033. https://doi.org/10.1590/2175-3369.011.e20190033

\section{Resumo}

Os serviços de mobilidade compartilhada (SMC) são uma alternativa inovadora de transportes nos centros urbanos. Sendo assim, utilizando-se de uma survey com 597 usuários, por meio de uma análise fatorial confirmatória, identificaram-se os determinantes do uso dos SMC no contexto brasileiro. Os benefícios econômicos, o prazer e a sustentabilidade são preditores da atitude e da intenção comportamental em relação aos SMC. Notou-se um relacionamento negativo entre reputação e atitude quanto aos SMC. Observou-se uma relação positiva entre atitude relativa aos SMC e intenção comportamental. Esses resultados contribuem metodologicamente para validar, no contexto brasileiro, uma escala de mensuração dos determinantes de adesão da utilização de SMC.

Palavras-chave: Mobilidade urbana. Serviços de mobilidade compartilhada. Determinantes. Análise fatorial confirmatória.

\begin{abstract}
Shared Mobility Services (SMC) is an innovative transportation alternative in urban centers. Thus, using a survey with 597 users, through a Confirmatory Factor Analysis, the determinants of SMC use in the Brazilian context was identified. Economic benefits, pleasure, and sustainability are predictors of Attitude toward SMCs and behavioral intent about SMCs. There was a negative relationship between Reputation and Attitude for the SMCs. A positive relationship was observed between Attitude and SMC and Behavioral Intent. These results contributed methodologically for validating a scale of measurement of the adherence determinants of the use of SMC in the Brazilian context.
\end{abstract}

Keywords: Urban mobility. Shared mobility services. Determinants. Confirmatory factorial analysis. 


\section{Introdução}

A mobilidade urbana tornou-se um problema para os centros urbanos (Moura, 2017), pois o aumento do poder aquisitivo e as políticas de incentivo à compra de automóveis (Cadurin, 2016) proporcionaram dependência dos transportes individuais, necessidade de deslocamento e congestionamentos (Mello, 2008), fatores que colaboram para a maior desumanização das cidades (Cadurin, 2016).

Nos grandes centros urbanos, há uma maior incidência de serviços de mobilidade compartilhada (SMC), que têm como fenômeno amplo a "economia compartilhada" (Le Vine \& Polak, 2015), contrário ao hiperconsumo (Freitas et al., 2016). A economia compartilhada adota práticas de mercado inteligentes para uma sociedade colaborativa e sustentável, por exemplo, o compartilhamento de bicicletas ou de carros (Heinrichs, 2013).

Embora existam estudos sobre as motivações e os fatores para a participação em serviços de compartilhamento (Hamari et al., 2016; Mohlmann, 2015; Yakin et al., 2017; Barbosa, 2017; Sampaio et al., 2018) e de mobilidade compartilhada (Murphy, 2016; Le Vine \& Polak, 2015; Shaheen et al., 2016), há uma lacuna relativa às pesquisas sobre o comportamento desses fatores em SMC no contexto brasileiro.

Neste estudo, a sustentabilidade, o prazer, a reputação, os benefícios econômicos e a atitude em relação aos SMC foram tratados como construtos exógenos, e a intenção comportamental, como construto endógeno. Este artigo sugere que os SMC buscam dar ou compartilhar acesso a produtos e serviços, de pessoa para pessoa, coordenados por meio de serviços on-line (Hamari et al., 2016).

O presente estudo mostra-se relevante por contribuir para o campo da pesquisa em SMC, vertente da "economia compartilhada", pouco investigada pela literatura, no contexto nacional. Os resultados incrementam o conhecimento sobre as motivações para utilização dos SMC no contexto da economia compartilhada, contribuindo para a introdução de novas considerações a respeito do perfil dos utilizadores e, sobretudo, dos fatores motivacionais que levam as pessoas a aderir a tal fenômeno.

Além desta Introdução, este artigo apresentará, na sequência, a fundamentação teórica, que embasa teoricamente o estudo. Depois, serão vistos os procedimentos metodológicos utilizados nesta investigação e os resultados obtidos. Por fim, serão apresentadas as considerações finais, as limitações e as indicações para futuras investigações.

\section{Sustentabilidade}

Um dos princípios da economia compartilhada é a sustentabilidade ecológica. Revender, trocar, alugar e emprestar a curto prazo, todas essas práticas entre indivíduos ou empresas fazem parte da economia de compartilhamento e têm o potencial de aumentar a duração do uso de bens que consomem recursos. Portanto, a participação no consumo colaborativo pode ser considerada ecologicamente sustentável (Demailly \& Novel, 2014).

Para Böcker \& Meelen (2017), embora as organizações de compartilhamento de acomodação de aluguel usem a sustentabilidade ambiental como um enquadramento motivacional para atrair novos clientes, seus usuários não se envolvem em compartilhamento de acomodação por motivos ambientais. Segundo Mohlmann (2015), o impacto ambiental não afeta o prazer ou a probabilidade de escolher o mesmo serviço de compartilhamento, uma vez que esse fator não compromete a intenção comportamental dos usuários dos serviços.

Conforme Hamari et al. (2016), a sustentabilidade percebida do serviço de compartilhamento associa-se à atitude em relação ao consumo colaborativo e não está relacionada às intenções comportamentais. Esse resultado corrobora a pesquisa de Yakin et al. (2017). Para Sampaio et al. (2018), a sustentabilidade não influencia positivamente a atitude em relação aos SMC e não está associada à intenção comportamental no que se refere à participação nos negócios de economia compartilhada. 
Conforme Miranda (2018), a sustentabilidade influencia positivamente a atitude de adesão dos consumidores de SMC. Para Mann (2018), o fator ambiental exerce uma influência na decisão dos indivíduos em utilizar os serviços de mobilidade compartilhada. Uma vez que os resultados da literatura não convergem e tendo em vista que as pesquisas no contexto nacional sinalizam a existência de relações positivas entre sustentabilidade e atitude dos consumidores quanto aos SMC (Miranda, 2018) e entre sustentabilidade e decisão de utilização do consumo de maneira compartilhada (Mann, 2018), formulam-se as duas primeiras hipóteses de pesquisa:

$\mathrm{H}_{1 \mathrm{a}}$ : A sustentabilidade influencia positivamente a atitude em relação aos serviços de mobilidade compartilhada.

$\mathrm{H}_{1 b}$ : A sustentabilidade influencia positivamente a intenção comportamental dos usuários de serviços de mobilidade compartilhada.

\section{Prazer}

O prazer é um aspecto fundamental da motivação intrínseca (Deci \& Ryan, 2000). Para Maurer et al. (2015), o prazer representa a segunda motivação intrínseca que leva as pessoas a participar da economia de compartilhamento.

Para muitos indivíduos, as razões econômicas são secundárias, uma vez que participam da economia compartilhada como forma de interação com a comunidade (Maurer et al., 2015). Esse novo modelo econômico proporciona experiências diferentes mediante o compartilhamento, permitindo que os participantes criem e mantenham conexões sociais significativas com outros indivíduos (Botsman \& Rogers, 2011). Para Hamari et al. (2016), o prazer influencia tanto a atitude das pessoas em participar das práticas de compartilhamento quanto a intenção comportamental. Resultados semelhantes foram observados por So et al. (2018) e por Sampaio et al. (2018). De acordo com Yang \& Ahn (2016), o prazer é um antecedente significativo da atitude em relação ao serviço do Airbnb.

Segundo Barbosa (2017), o prazer foi valorizado entre os usuários de acomodação P2P e está diretamente relacionado ao valor da experiência (motivação intrínseca) e ao desempenho das plataformas (motivação extrínseca). Já Tussyadiah \& Pesonen (2018) identificaram que o prazer foi o elo mais forte entre intenção e prazer em plataformas P2P. Assim, suportado pelos resultados reportados na literatura, propõem-se as seguintes hipóteses de pesquisa:

$\mathrm{H}_{2 \mathrm{a}}$ : $\mathrm{O}$ prazer percebido influencia positivamente a atitude em relação aos serviços de mobilidade compartilhada.

$\mathrm{H}_{2 \mathrm{~b}}$ : 0 prazer percebido influencia positivamente a intenção comportamental dos usuários de serviços de mobilidade compartilhada.

\section{Reputação}

A reputação é o caráter de certa marca ou, ainda, a percepção geral das particularidades substanciais sobre empresas ou marcas (Fombrun \& Rindova, 2000). Na visão de Liang \& Shi (2005), a reputação é o julgamento de valor por parte de um avaliador a partir do comportamento passado do avaliado. Assim, a reputação seria um fator importante de motivação externa para a participação no consumo colaborativo (Maurer et al., 2015), uma vez que pode estar relacionada à confiança (Kim et al., 2015).

Segundo Hamari et al. (2016), a reputação afeta a atitude das pessoas em relação às atividades compartilhadas e a intenção comportamental, resultado que foi evidenciado por Yakin et al. (2017). Já Sampaio et al. (2018) notaram que, no contexto brasileiro, a reputação associa-se positivamente à atitude quanto aos SMC, muito embora não tenham sido notados indícios empíricos de que esteja relacionada à intenção comportamental dos indivíduos em participar dos negócios de compartilhamento. Segundo Yang \& Ahn (2016), a reputação é um antecedente significativo de atitude em relação ao Airbnb, 
pois tende a ser um impulsionador pessoal dos tipos de comportamento do consumidor. Assim, com base nos resultados empíricos mapeados na literatura, propõem-se as seguintes hipóteses de pesquisa:

$\mathrm{H}_{3 \mathrm{a}}$ : A reputação percebida influencia positivamente a atitude em relação aos serviços de mobilidade compartilhada.

$\mathrm{H}_{3 \mathrm{~b}}$ : A reputação percebida influencia positivamente a intenção comportamental dos usuários de serviços de mobilidade compartilhada.

\section{Benefícios econômicos}

A participação na economia de compartilhamento demonstra um comportamento racional de maximização da utilidade (Maurer et al., 2015) pela substituição da propriedade exclusiva de bens por um serviço de compartilhamento de baixo custo (Hamari et al., 2016).

Hamari et al. (2016) notaram que os benefícios econômicos não apresentam efeito significativo na atitude em relação aos SMC, no entanto exercem influência direta e positiva na intenção comportamental de participar das práticas de compartilhamento. Por sua vez, Yakin et al. (2017) observaram que os benefícios econômicos têm uma relação positiva com a atitude e associam-se negativamente à intenção comportamental. Já Sampaio etal. (2018) observaram que os benefícios econômicos impactam positivamente na atitude quanto aos SMC e na intenção comportamental. Para Pinotti (2016), os benefícios econômicos influenciam a intenção de recompra dos usuários dos meios de hospedagem alternativos, uma vez que demonstram uma maior preocupação com a experiência em si. Quanto a Mann (2018), ele notou que os benefícios econômicos percebidos exercem uma influência sobre a atitude dos consumidores em utilizar o consumo compartilhado.

Tussyadiah \& Pesonen (2018) identificaram que os benefícios econômicos estão associados à intenção de uso desses modelos de acomodações, principalmente entre os viajantes jovens. Portanto, a partir dos resultados empíricos observados, sugerem-se as seguintes hipóteses de pesquisa:

$\mathrm{H}_{4 a}$ : Os benefícios econômicos percebidos influenciam positivamente a atitude em relação aos serviços de mobilidade compartilhada.

$\mathrm{H}_{4 b}$ : Os benefícios econômicos percebidos influenciam positivamente a intenção comportamental dos usuários de serviços de mobilidade compartilhada.

\section{Atitude em relação aos SMC e intenção comportamental}

A atitude é um fator determinante das intenções comportamentais, uma vez que exprime os gostos e as aversões dos indivíduos (Engel et al., 2000), e é um importante antecedente do comportamento (Ajzen, 1991; Engel et al., 2000; Wang \& Liu, 2009). De acordo com a teoria da ação racional (Fishbein, 1963, 1967), as atitudes e as intenções precedem o comportamento e estão relacionadas a um conjunto de fatores pessoais e sociais (normas subjetivas). Para Lopes \& Alves (2017), a atitude é um fator interacional dinâmico em que um indivíduo se correlaciona com o objeto da atitude e que pode ser alterado ao longo do tempo.

A atitude influencia a intenção de compra futura, manifestada mediante um comportamento individual direcionado para um produto (Wang \& Yang, 2008). Esse comportamento representa um reflexo da afetividade, que tem influência no comportamento do consumidor (Hultman et al., 2015). Assim, a intenção de comportamento é a disposição ou o planejamento dos indivíduos em executar um comportamento particular (Ajzen, 1991).

Hamari et al. (2016) identificaram que a atitude quanto aos SMC pode influenciar as intenções comportamentais, no entanto isso ocorre em um grau muito pequeno em comparação à relação tipicamente observada entre esses construtos. Esse resultado também foi observado nas pesquisas de Yakin et al. (2017) e Sampaio et. al. (2018). Partindo do pressuposto de que a atitude seria uma 
informação ou uma avaliação sobre determinados produtos e serviços (Fishbein \& Ajzen, 1975; Bagozzi \& Burnkrant, 1979), consumidores que adquirem informações e opiniões do seu grupo de referência têm uma intenção mais forte para comprar determinados produtos do que os que não têm informações ou opiniões sobre um produto específico (Conner et al., 2001).

Para este estudo, a atitude em relação aos SMC caracteriza-se como: (1) um dos principais determinantes do comportamento; (2) distinta da ação, isto é, do comportamento efetivo; e (3) relacionada à motivação para participar ou consumir. Diante do exposto, propõe-se a última hipótese da pesquisa:

$\mathrm{H}_{5}$ : A atitude em relação aos SMC influencia positivamente a intenção comportamental dos usuários de serviços de mobilidade compartilhada.

Conforme a Figura 1, o modelo proposto apresenta dois construtos endógenos (intenção comportamental e atitude) e quatro construtos exógenos (sustentabilidade, prazer, reputação e benefícios econômicos), além duas variáveis de controle (gênero e renda).

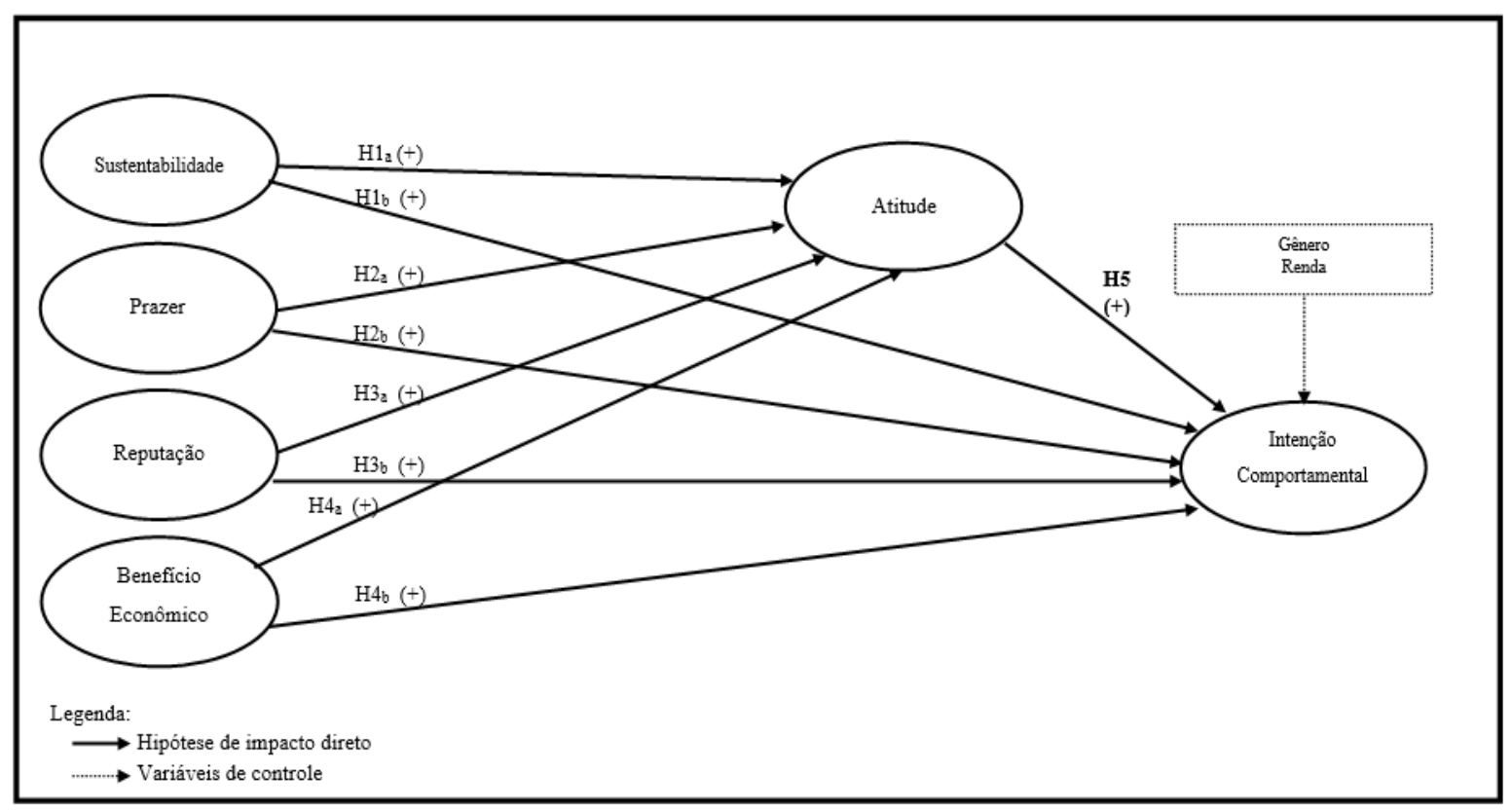

Figura 1 - Modelo conceitual investigado. Fonte: adaptada de Hamari et al. (2016).

\section{Método da pesquisa}

Foram investigados 597 utilizadores de serviços, como o compartilhamento de carros, de caronas ou de bicicletas, existentes no Brasil. A escolha pelos SMC justifica-se por serem os segmentos da economia compartilhada que mais crescem no país (Freese et al., 2014). Utilizou-se do instrumento de coleta proposto por Hamari et al. (2016), com a inserção do termo consumo colaborativo para SMC, uma vez que a escala original identifica os fatores determinantes para participação nas atividades de compartilhamento de forma genérica.

Foi utilizado o modelo de Hamari et al. (2016) composto de atitude, intenção comportamental, prazer, benefício econômico, reputação e sustentabilidade, mensurados por uma escala Likert de 5 pontos (em que 1 corresponde a "discordo totalmente", e 5, "concordo totalmente"). Foi empregada a ferramenta Google Docs para a coleta de dados, sendo os questionários enviados e multiplicados, de 29 de junho de 2018 a 6 de agosto de 2018, via postagens em redes sociais (Facebook), seguindo o método conhecido como "bola de neve" (Malhotra, 2012). 0 Quadro 1 apresenta a escala dos construtos da pesquisa: 
Quadro 1 - Questionário adaptado para a pesquisa

\begin{tabular}{|c|c|}
\hline Item & Descrição \\
\hline \multicolumn{2}{|r|}{ Dimensão atitude } \\
\hline AT_1 & Acho que utilizar os serviços de mobilidade compartilhada é uma medida inteligente. \\
\hline AT_2 & Acho que os serviços de mobilidade compartilhada são positivos. \\
\hline AT_3 & Acho que os serviços de mobilidade compartilhada são bons. \\
\hline AT_4 & No geral, a utilização dos serviços de mobilidade compartilhada dentro de uma comunidade faz sentido. \\
\hline \multicolumn{2}{|r|}{ Dimensão intenção comportamental } \\
\hline IC_l & Os serviços de mobilidade compartilhada são melhores do que as práticas de mobilidade tradicionais. \\
\hline IC_2 & Considerando tudo, espero utilizar os serviços de mobilidade compartilhada com frequência no futuro. \\
\hline IC_3 & Eu me vejo utilizando com mais frequência os serviços de mobilidade compartilhada no futuro. \\
\hline IC_4 & Se for possível, eu me vejo utilizando os serviços de mobilidade compartilhada. \\
\hline IC_5 & É provável que eu utilize frequentemente os serviços de mobilidade compartilhada no futuro. \\
\hline \multicolumn{2}{|r|}{ Dimensão prazer } \\
\hline PRA_1 & Eu acho que os serviços de mobilidade compartilhada são agradáveis. \\
\hline PRA_2 & Eu acho que os serviços de mobilidade compartilhada são empolgantes. \\
\hline PRA_3 & Eu acho que os serviços de mobilidade compartilhada são divertidos. \\
\hline PRA_4 & Eu acho que os serviços de mobilidade compartilhada são interessantes. \\
\hline \multicolumn{2}{|r|}{ Dimensão benefícios econômicos } \\
\hline ECO_1 & Eu posso poupar dinheiro utilizando os serviços de mobilidade compartilhada. \\
\hline ECO_2 & A utilização dos serviços de mobilidade compartilhada me beneficia em termos financeiros. \\
\hline ECO_3 & Os serviços de mobilidade compartilhada permitem melhorar minha situação financeira. \\
\hline ECO_4 & Os serviços de mobilidade compartilhada permitem-me poupar tempo. \\
\hline \multicolumn{2}{|r|}{ Dimensão reputação } \\
\hline REP_1 & Utilizar os serviços de mobilidade compartilhada melhora minha imagem na comunidade. \\
\hline REP_2 & Ganho reconhecimento por utilizar os serviços de mobilidade compartilhada. \\
\hline REP_3 & Ganharia o respeito dos outros utilizando os serviços de mobilidade compartilhada. \\
\hline REP_4 & $\begin{array}{l}\text { As pessoas da comunidade que utilizam os serviços de mobilidade compartilhada têm mais prestígio do } \\
\text { que as que não utilizam. }\end{array}$ \\
\hline \multicolumn{2}{|r|}{ Dimensão sustentabilidade } \\
\hline SUST_1 & Os serviços de mobilidade compartilhada ajudam a preservar os recursos naturais. \\
\hline SUST_2 & Os serviços de mobilidade compartilhada mostram-se como uma forma sustentável de consumo. \\
\hline SUST_3 & Os serviços de mobilidade compartilhada são modelos de transporte ecológico. \\
\hline SUST_4 & Os serviços de mobilidade compartilhada são modelos de transporte eficiente em termos de uso de energia. \\
\hline SUST_5 & Os serviços de mobilidade compartilhada são modelos de transporte ecologicamente corretos. \\
\hline
\end{tabular}

Fonte: Adaptado de Hamari et al. (2016).

Realizou-se um pré-teste, por meio de uma amostra composta de 92 respondentes, para averiguar a consistência interna dos construtos utilizados no estudo. Após verificar a confiabilidade das escalas (alfa de Cronbach de 0,87 para atitude em relação aos SMC; 0,91 para intenção comportamental; 0,86 para prazer; 0,74 para benefícios econômicos; 0,93 para reputação; e 0,93 para sustentabilidade) e os ajustes semânticos, utilizou-se de uma análise fatorial exploratória (AFE). 0 teste final foi realizado por meio de um questionário hospedado no Google Forms. Empregou-se a técnica estatística da modelagem de equações estruturais (MEE), ou Structural Equation Modeling (SEM), na sua abordagem fundamentada na covariância (Covariance-Based Structural Equation Modeling - CB-SEM). Com o auxílio do software IBM SPSS Amos $^{\circledR}$, versão 20.0, realizou-se a etapa inicial da análise CB-SEM, com a análise fatorial confirmatória (AFC) para avaliar a qualidade do ajuste do modelo de mensuração teórico quanto à estrutura correlacional observada entre conjunto de variáveis observadas (Maroco, 2010). Após assegurada a qualidade do ajuste dos dados, passou-se para a avaliação das relações estruturais por meio da segunda fase da abordagem CB-SEM.

\section{Análise e discussão dos dados}

Neste tópico, serão apresentados e discutidos os resultados obtidos no estudo. 0 respondente médio da amostra pode ser caracterizado como do gênero feminino, com idade média de 26 anos, detendo nível superior incompleto, renda familiar de 4,69 salários mínimos, residente da região Sul/Sudeste e que utiliza os serviços do aplicativo Uber $(88,61 \%)$, sendo que $45 \%$ utilizam exclusivamente esse serviço. 


\section{Avaliação preliminar dos construtos}

De forma a avaliar a existência do alcance dos pressupostos inerentes à condução da análise CB-SEM, avaliou-se, construto a construto, a existência de correlação entre os itens, bem como o nível de consistência interna das escalas (via alfa de Cronbach). Os resultados obtidos possibilitam concluir a ausência de problemas de multicolinearidade dos itens, sendo notada a existência de excelentes níveis de consistência interna (alfa de Cronbach superiores a 0,80).

\section{Análise fatorial confirmatória}

$\mathrm{Na}$ análise inicial, o item ECO_4 ("Os serviços de mobilidade compartilhada permitem-me poupar tempo") apresentou baixa carga fatorial padronizada $(\beta=0,16)$; assim, optou-se por excluir esse item da análise, tendo em vista que a sua manutenção acarretava a obtenção de baixos índices de qualidade do ajuste (goodness of fit - GOF). Como possível explicação para a não relevância dessa variável no contexto analisado, pode-se supor que seja reflexo dos constantes congestionamentos presentes nas grandes metrópoles, da baixa infraestrutura de ciclovias, dentre outros fatores possíveis. Dessa forma, mais estudos são necessários para incrementar a compreensão desse resultado.

Na sequência, avaliou-se a qualidade do ajuste do modelo (goodness of fit - GOF), que informa o quanto o modelo reproduz a matriz de covariância entre os indicadores, ou seja, as matrizes de covariância estimada e observada (Hair et al., 2016). Assim, os valores reportados $(\chi 2 / \mathrm{Gl}=2,94$; GFI = 0,91; $\mathrm{NFI}=0,94 ; \mathrm{RFI}=0,93 ; \mathrm{IFI}=0,96 ; \mathrm{CFI}=0,96$; $\mathrm{TLI}=0,95 ; \mathrm{PCFI}=0,81$; $\mathrm{RMSEA}=0,06$ ) revelaram que o modelo apresenta excelentes níveis de ajustamentos, uma vez que todos atendem aos limites preconizados na literatura, isto é, $\chi 2 /$ Gl obtido é inferior a 5; o RMSEA é menor que 0,08 ; os valores de GFI, NFI, RFI, IFI, CFI e TLI são suficientemente inferiores a 0,90; e o valor de PCFI está entre 0,06 e 0,8 (Hair et al., 2016).

O índice de parcimônia de ajustamento (PRATIO $=0,08$ ) do modelo proposto é considerado satisfatório, uma vez que apresenta valor superior 0,06, reportado na literatura (Ribas \& Vieira, 2011). Foram notados valores inferiores (inclusive para os indicadores ECVI, LO 90, HI 90 e MECVI) para o modelo hipotético, quando comparado aos modelos saturados e independentes, pelo critério de informação de Akaike (AIC), critério Browne-Cudeck (BCC), critério de informação Bayes (BIC) e critério de informação de Akaike consistente (CAIC). Conclui-se que o modelo teórico apresentou o melhor ajustamento. As estimativas dos pesos de regressão demonstraram razões críticas superiores a 1,96 (CR $>1,96)$ e nível de significância estatística superior a $5 \%(p<0,05)$. Desse modo, notou-se que, entre os indicadores dos construtos, há uma covariância sistemática e estatisticamente significante.

Um valor de AVE superior a 0,50 indica que um construto explica metade da variância de seus indicadores (Hair etal., 2016). Observou-se que todos indicadores das variáveis latentes (construtos) reportaram valores de AVE acima do mínimo recomendado (de 0,50), indicando a existência de nível satisfatório de validade convergente.

Os valores reportados para a confiabilidade em cada construto (atitude $=0,91$; benefícios econômicos $=0,80$; sustentabilidade $=0,91$; intenção comportamental $=0,89$; reputação $=0,92$; $\mathrm{e}$ satisfação $=0,85$ ) mostraram-se suficientemente superiores a 0,70 , valor preconizado pela literatura. Portanto, não ocorreram problemas de validade convergente. Acerca da validade discriminante, analisada por meio do critério proposto por Fornell \& Larcker (1981), notou-se que nenhuma das correlações entre os construtos mostrou-se superior à raiz quadrada da AVE. Assim, conclui-se pela existência de validade discriminante em cada construto do modelo investigado.

Conforme a Figura 2, o coeficiente de determinação $\left(\mathrm{R}^{2}\right)$ identifica a porção da variância do construto endógeno (independente) explicada pelos construtos exógenos (dependentes) a ele relacionados (Hair et al., 2016). De acordo com Ringle et al. (2014, p. 7), o coeficiente de determinação (R²) “[...] indica 
a qualidade do modelo ajustado", em que os valores $0,75,0,50$ e 0,25 são considerados, respectivamente, substanciais, moderados e fracos (Hair et al., 2016).

Cerca de $56 \%$ da variância do construto atitude em relação aos SMC $\left(\mathrm{R}^{2}=0,56\right)$ e $66 \%$ da variância do construto intenção comportamental $\left(\mathrm{R}^{2}=0,66\right)$ foram explicadas pelo conjunto de variáveis independentes (e de controle, isto é, gênero e renda) existentes no modelo estrutural. Conforme Hair et al. (2016), conclui-se que ambos os construtos são explicados moderadamente.

Após isolar o efeito das variáveis de controle gênero e renda, conclui-se que os benefícios econômicos estão positivamente relacionados à atitude em relação aos SMC $(\beta=0,112 ; p<0,001)$, confirmando a hipótese 1a. Esse resultado corrobora os estudos de Miranda (2018), So et al. (2018), Yakin et al. (2017) e Sampaio et al. (2018) e contrariam os achados de Hamari et al. (2016), justificando-se pelas dificuldades financeiras dos indivíduos (cerca de $2 / 3$ da amostra apontou ter renda de até 5 salários mínimos, sendo que $32 \%$ do total relatou ganhar até 1 salário mínimo), indicando que a atitude em relação aos SMC é motivada pelos benefícios econômicos que os usuários podem ter.

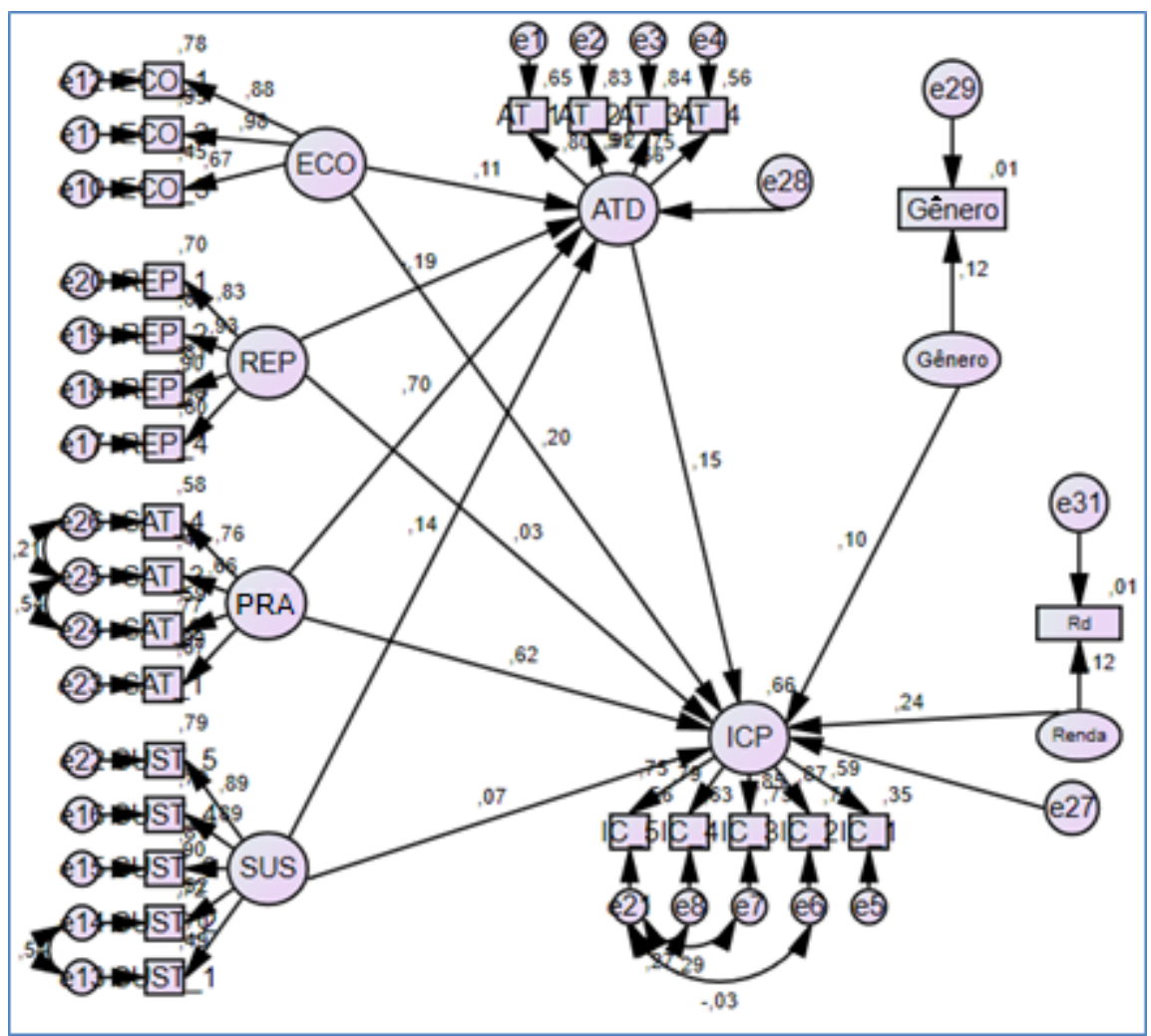

Figura 2 - Resultados da análise do modelo estrutural. Fonte: Dados da pesquisa (2018).

Tendo em vista a existência, para este estudo, de uma relação positiva entre os benefícios econômicos e a intenção comportamental $(\beta=0,20 ; p<0,001)$, a hipótese $1 \mathrm{~b}$ foi confirmada. No entanto, esse resultado não se alinha aos achados de Yakin et al. (2017). Por outro lado, eles são coerentes com os estudos de Hamari etal. (2016) e de Sampaio etal. (2018), que demonstram que os benefícios econômicos são fatores influenciadores da intenção de utilização dos SMC, uma vez que, ao optar por utilizar esse tipo de serviço, o usuário teria a expectativa de obter uma economia financeira por não ter custos relacionados à reparação e à manutenção, já que não é proprietário dos bens (Moeller \& Wittkowski, 2010).

A reputação está relacionada negativamente com a atitude em relação aos SMC $(\beta=-0,19 ; p<0,001)$, refutando-se, assim, a hipótese 2. Esse resultado corrobora os resultados de Hamari et al. (2016), Yang \& Ahn (2016) e Sampaio et al. (2018). A utilização desse tipo de serviço de compartilhamento não melhora a imagem do usuário diante da comunidade nem o faz ganhar respeito, reconhecimento e/ou prestígio. 
Quanto à hipótese que evidencia uma relação positiva entre reputação e intenção comportamental, observou-se uma relação não significante $(\beta=0,03 ; p<$ não significante). Portanto, a hipótese $2 b$ não se confirmou. Esse resultado não se alinha aos achados de Yakin et al. (2017), contudo corrobora os estudos de Hamari et al. (2016) e Sampaio et al. (2018), uma vez que o ganho de reputação não repercute em mais consumo de atividades de compartilhamento. Esses insights demandam uma análise multigrupo, considerando as variáveis de controle (renda e gênero). Observou-se uma relação positiva entre prazer e atitude em relação aos SMC $(\beta=0,70 ; \mathrm{p}<0,001)$, confirmando, assim, a hipótese 3a. Esse resultado está alinhado aos obtidos por Hamari et al. (2016), So et al. (2018), Yang \& Ahn (2016) e Sampaio et al. (2018). Os indivíduos perceberam os SMC como potencialmente agradáveis, divertidos, interessantes e empolgantes. Foi evidenciada a relação positiva entre prazer e intenção comportamental $(\beta=0,62$; $\mathrm{p}<0,001$ ), confirmando, dessa maneira, a hipótese $3 \mathrm{~b}$, resultado que está em consonância com as pesquisas Hamari et al. (2016), Yakin et al. (2017) e Sampaio et al. (2018).

Notou-se que a sustentabilidade se relaciona positivamente com a atitude em relação aos SMC $(\beta=0,14 ; p<0,001)$, confirmando a hipótese 4a. Esse resultado contraria Sampaio et al. (2018), mas essa relação mapeada no presente estudo é respaldada pela literatura e pode ser observada nas pesquisas de Hamari et al. (2016) e de Miranda (2018). Na amostra analisada, as atividades de compartilhamento acomodam necessidades e desejos de maneira mais sustentável com menores danos ao meio ambiente (Pizzol, 2015), quando comparadas às alternativas tradicionais de mobilidade; assim, também foi confirmada a hipótese $4 \mathrm{~b}$, da relação positiva entre sustentabilidade e intenção comportamental $(\beta=0,97 ; p<0,001)$. Porém, esse resultado não corrobora os estudos de Sampaio et al. (2018) e Mohlmann (2015), mas encontra respaldo em Hamari et al. (2016) e Yakin et al. (2017). Ao contrário das descobertas da pesquisa de Sampaio et al. (2018), os achados indicam que, para os usuários dos serviços de mobilidade compartilhada, a consciência ambiental e a sustentabilidade são variáveis que estão diretamente relacionadas com possíveis novas oportunidades geradas pelo compartilhamento.

$\mathrm{Na}$ busca de evidenciar a relação positiva entre atitude em relação aos SMC e intenção comportamental, evidenciou-se, pelos resultados reportados, a confirmação da hipótese $5(\beta=0,15$; $\mathrm{p}<0,001)$. É importante ressaltar que as variáveis de controle inseridas no modelo estrutural se mostraram positivamente relacionadas; logo, são obtidos indícios empíricos da existência de um padrão de respostas distintos por gênero $(\beta=0,10 ; p<0,01)$, indicando que, em média, as mulheres apresentam maiores níveis de intenção comportamental e renda $(\beta=0,24 ; p<0,01)$, o que sinaliza que os grupos de renda mais elevada apresentam maior intenção comportamental.

Os resultados obtidos apontam a relevância de benefícios econômicos, prazer e sustentabilidade como "preditores" que influenciam diretamente a atitude em relação aos SMC e a intenção comportamental dos usuários do presente estudo que utilizam os SMC. Acerca da reputação, foram notadas uma influência significativa na atitude em relação aos $\operatorname{SMC}(\beta=-0,19 ; \mathrm{p}<0,001)$ e uma relação não significativa quanto à intenção comportamental $(\beta=0,03 ; p<$ não significante).

De forma a consolidar os achados empíricos do presente estudo e possibilitar a sua confrontação com a literatura empírica correlata, reporta-se o Quadro 2. 
Quadro 2 - Confrontação da presente pesquisa com a literatura correlata

\begin{tabular}{|c|c|c|c|}
\hline Identificação & Objetivos do estudo & Metodologia & Resultados \\
\hline $\begin{array}{l}\text { Hamari et al. } \\
\text { (2016) }\end{array}$ & $\begin{array}{l}\text { Investigar as } \\
\text { motivações das } \\
\text { pessoas para } \\
\text { participar do } \\
\text { consumo } \\
\text { colaborativo. }\end{array}$ & $\begin{array}{l}\text { Survey respondido por } 168 \text { usuários } \\
\text { registrados no serviço Sharetribe e } \\
\text { que foram recrutados por meio de } \\
\text { um boletim informativo oficial do } \\
\text { Sharetribe por e-mail. Os dados } \\
\text { demográficos descritivos foram } \\
\text { analisados com o SPSS 20, e todos } \\
\text { os testes foram conduzidos por } \\
\text { mínimos quadrados parciais (PLS), e } \\
\text { a análise foi feita com o SmartPLS } \\
2,0 \mathrm{M} 3 \text {. }\end{array}$ & $\begin{array}{l}\text { A sustentabilidade percebida do serviço } \\
\text { de compartilhamento está associada } \\
\text { positivamente à atitude em relação ao } \\
\text { consumo colaborativo, não apresentando } \\
\text { efeito direto sobre as intenções } \\
\text { comportamentais. } \\
\text { O prazer exerce efeito significativo tanto } \\
\text { na atitude das pessoas em participar das } \\
\text { práticas de compartilhamento quanto na } \\
\text { intenção comportamental. } \\
\text { A reputação afeta de maneira } \\
\text { significativa a atitude das pessoas em } \\
\text { relação às atividades compartilhadas, } \\
\text { bem como a intenção comportamental. } \\
\text { Os benefícios econômicos exercem } \\
\text { influência direta e positiva na intenção } \\
\text { comportamental de participar das } \\
\text { práticas de compartilhamento. } \\
\text { Quanto à relação empírica entre atitude e } \\
\text { intenção comportamental, identificou-se } \\
\text { que a atitude em relação aos SMC exerce } \\
\text { uma influência direta e positiva nas } \\
\text { intenções comportamentais. }\end{array}$ \\
\hline $\begin{array}{l}\text { Mohlmann } \\
\text { (2015) }\end{array}$ & $\begin{array}{l}\text { Identificar os } \\
\text { determinantes mais } \\
\text { importantes para } \\
\text { explicar a satisfação } \\
\text { de escolher uma } \\
\text { opção de } \\
\text { compartilhamento e } \\
\text { identificar os } \\
\text { determinantes mais } \\
\text { importantes para } \\
\text { explicar a } \\
\text { probabilidade de } \\
\text { escolher uma opção } \\
\text { de } \\
\text { compartilhamento } \\
\text { novamente. }\end{array}$ & $\begin{array}{l}\text { A coleta dos dados ocorreu por } \\
\text { meio de dois estudos on-line } \\
\text { quantitativos independentes, } \\
\text { distribuídos sequencialmente por } \\
\text { meio de uma lista de discussão para } \\
\text { estudantes da Universidade de } \\
\text { Hamburgo: no estudo 1, foram } \\
\text { pesquisados os usuários do serviço } \\
\text { de compartilhamento de carros B2C } \\
\text { Car2Go; e no estudo } 2 \text {, foram } \\
\text { pesquisados os usuários do mercado } \\
\text { de hospedagem comunitária on-line } \\
\text { C2C Airbnb. } \\
\text { A modelagem de caminho de } \\
\text { mínimos quadrados parciais (PLS) } \\
\text { com base em variância técnica foi } \\
\text { aplicada para testar as hipóteses } \\
\text { utilizando o software SmartPLS 2.0. }\end{array}$ & $\begin{array}{l}\text { Foram constatadas a satisfação e a } \\
\text { probabilidade de escolher uma opção de } \\
\text { compartilhamento novamente para ser } \\
\text { explicada predominantemente por } \\
\text { determinantes que servem de benefício } \\
\text { próprio aos usuários. Utilidade, confiança, } \\
\text { economia de custos e familiaridade foram } \\
\text { consideradas essenciais em ambos os } \\
\text { estudos, enquanto a qualidade do serviço } \\
\text { e o pertencimento à comunidade foram } \\
\text { identificados somente no estudo } 1 .\end{array}$ \\
\hline $\begin{array}{l}\text { Yakin et al. } \\
\text { (2017) }\end{array}$ & $\begin{array}{l}\text { Determinar a } \\
\text { motivação de } \\
\text { participação dos } \\
\text { consumidores turcos } \\
\text { que contribuem } \\
\text { para a economia } \\
\text { de partilha, } \\
\text { utilizando a teoria da } \\
\text { autodeterminação } \\
\end{array}$ & $\begin{array}{l}\text { O estudo empregou a escala } \\
\text { utilizada por Hamari et al. (2016). } \\
\text { A análise fatorial confirmatória foi } \\
\text { realizada primeiramente usando o } \\
\text { programa Amos } 22 \text { e, em seguida, a } \\
\text { modelagem de equações } \\
\text { estruturais. As hipóteses foram } \\
\text { testadas para verificar fatores de } \\
\text { escala no estudo. }\end{array}$ & $\begin{array}{l}\text { Entre os três fatores motivacionais } \\
\text { (benefício econômico, prazer e } \\
\text { sustentabilidade), apenas o nível de prazer } \\
\text { percebido tem o maior efeito sobre a } \\
\text { atitude e a intenção do comportamento. }\end{array}$ \\
\hline $\begin{array}{c}\text { Yang \& Ahn } \\
(2016)\end{array}$ & $\begin{array}{l}\text { Verificar como a } \\
\text { motivação para a } \\
\text { economia } \\
\text { compartilhada e a } \\
\text { segurança } \\
\text { percebida afetam a } \\
\text { atitude e a lealdade } \\
\text { dos usuários em } \\
\text { relação a um } \\
\text { negócio } \\
\text { representativo de } \\
\text { economia } \\
\text { compartilhada, o } \\
\text { Airbnb. }\end{array}$ & $\begin{array}{l}\text { Os resultados foram obtidos por } \\
\text { meio uma pesquisa de } \\
\text { direcionamento de URL, projetada } \\
\text { para trabalhar em dispositivos } \\
\text { móveis, sendo o público } \\
\text { investigado estudantes universitários } \\
\text { que fizessem cursos relacionados ao } \\
\text { marketing. Para análise dos dados, } \\
\text { foi empregada a técnica de } \\
\text { modelagem de equações } \\
\text { estruturais (SEM). }\end{array}$ & $\begin{array}{l}\text { O prazer e a reputação tiveram um efeito } \\
\text { positivo na atitude em relação à Airbnb, } \\
\text { enquanto outros aspectos motivacionais, } \\
\text { como sustentabilidade e benefícios } \\
\text { econômicos, pareciam não ter nenhum } \\
\text { efeito significativo. }\end{array}$ \\
\hline
\end{tabular}


Quadro 2 - Continuação...

\begin{tabular}{|c|c|c|c|}
\hline Identificação & Objetivos do estudo & Metodologia & Resultados \\
\hline So et al. (2018) & $\begin{array}{l}\text { Examinar o poder } \\
\text { preditivo das } \\
\text { motivações e das } \\
\text { restrições que } \\
\text { afetam a adoção } \\
\text { pelo consumidor do } \\
\text { Airbnb. }\end{array}$ & $\begin{array}{l}\text { Pesquisa on-line nacional e } \\
\text { quantitativa nos Estados Unidos, } \\
\text { medindo as motivações e as } \\
\text { restrições dos consumidores da } \\
\text { Airbnb para usar a acomodação } \\
\text { do Airbnb. A coleta de dados foi } \\
\text { por meio do Qualtrics Online } \\
\text { Sample. } \\
\text { O poder preditivo dos fatores de } \\
\text { motivação e de restrição foi } \\
\text { testado pela modelagem dos } \\
\text { mínimos quadrados parciais } \\
\text { (PLS-PM). }\end{array}$ & $\begin{array}{l}\text { Quanto às motivações, o valor do preço, } \\
\text { o prazer e os benefícios domésticos } \\
\text { explicam significativamente a atitude } \\
\text { geral em relação ao Airbnb. Quanto às } \\
\text { restrições, a desconfiança é o único fator } \\
\text { que prevê significativamente a atitude } \\
\text { geral, enquanto a insegurança está } \\
\text { diretamente relacionada às intenções } \\
\text { comportamentais. Atitude geral, controle } \\
\text { comportamental percebido e normas } \\
\text { subjetivas, como influência social, } \\
\text { tendência e afinidade, são preditores de } \\
\text { intenções comportamentais. }\end{array}$ \\
\hline Miranda (2018) & $\begin{array}{l}\text { Identificar os fatores } \\
\text { que estão } \\
\text { associados à atitude } \\
\text { em relação ao } \\
\text { consumo } \\
\text { colaborativo no } \\
\text { Brasil }\end{array}$ & $\begin{array}{l}\text { A amostra foi composta de } \\
321 \text { respondentes. Realizou-se uma } \\
\text { regressão linear múltipla para } \\
\text { verificar a associação da variável } \\
\text { dependente. }\end{array}$ & $\begin{array}{l}\text { Os benefícios econômicos, a } \\
\text { sustentabilidade e a dimensão materialista } \\
\text { da satisfação dos respondentes possuíam } \\
\text { tendência a se associar positivamente } \\
\text { com a atitude em relação ao consumo } \\
\text { colaborativo, enquanto os riscos } \\
\text { percebidos representaram uma barreira à } \\
\text { prática do consumo colaborativo. }\end{array}$ \\
\hline $\begin{array}{c}\text { Sampaio et al. } \\
\text { (2018) }\end{array}$ & $\begin{array}{l}\text { Demonstrar o } \\
\text { comportamento do } \\
\text { consumidor no } \\
\text { modelo de } \\
\text { economia } \\
\text { compartilhada, } \\
\text { possibilitando } \\
\text { compreender como } \\
\text { ele interage e quais } \\
\text { são as suas } \\
\text { expectativas. }\end{array}$ & $\begin{array}{l}\text { O instrumento de pesquisa foi } \\
\text { construído a partir do modelo } \\
\text { proposto por Hamari et al. (2016), } \\
\text { montado na plataforma Google } \\
\text { Forms e distribuído pelas redes } \\
\text { sociais, de forma a obter a maior } \\
\text { abrangência possível na região } \\
\text { citada. } \\
\text { Para análise dos dados, foi } \\
\text { empregada a técnica de } \\
\text { modelagem de equações } \\
\text { estruturais (SEM). Os constructos } \\
\text { foram submetidos à análise fatorial } \\
\text { confirmatória, utilizando-se do } \\
\text { software SmartPLS 3.2. }\end{array}$ & $\begin{array}{l}\text { Ao analisar as motivações intrínsecas, a } \\
\text { variável sustentabilidade não foi capaz de } \\
\text { prever significativamente a atitude para } \\
\text { CC e tampouco teve influência direta ou } \\
\text { indireta nas intenções de comportamento } \\
\text { para CC. A variável satisfação percebida } \\
\text { foi positivamente associada à atitude em } \\
\text { relação ao CC e à intenção de } \\
\text { comportamento na participação do } \\
\text { consumo colaborativo. A análise das } \\
\text { motivações extrínsecas evidenciou que o } \\
\text { possível aumento na reputação não } \\
\text { causa influência nas atitudes em relação } \\
\text { ao CC, como também não está } \\
\text { associado à intenção comportamental no } \\
\text { que tange à participação do consumo } \\
\text { colaborativo. Ainda nas motivações } \\
\text { extrínsecas, em contraposição ao } \\
\text { observado na variável reputação, as } \\
\text { motivações relativas a benefícios } \\
\text { econômicos foram positivamente } \\
\text { associadas a atitudes em relação ao } \\
\text { consumo colaborativo e tiveram influência } \\
\text { significativamente positiva na intenção } \\
\text { comportamental de participar do CC. } \\
\text { Por fim, a atitude quanto ao consumo } \\
\text { colaborativo teve efeito positivo sobre a } \\
\text { intenção comportamental da } \\
\text { participação do CC. }\end{array}$ \\
\hline Presente estudo & $\begin{array}{l}\text { Identificar os } \\
\text { determinantes da } \\
\text { utilização dos SMC } \\
\text { no contexto } \\
\text { brasileiro }\end{array}$ & $\begin{array}{l}\text { Realizou-se uma survey com } \\
597 \text { respondentes, utilizadores dos } \\
\text { SMC, tendo como base o } \\
\text { instrumento de pesquisa de } \\
\text { Hamari et al. (2016), com pontuais } \\
\text { adaptações semânticas. Como } \\
\text { técnica multivariada da análise de } \\
\text { dados, utilizou-se da análise fatorial } \\
\text { confirmatória (Covariance-Based } \\
\text { Structural Equation Modeling - } \\
\text { CB-SEM). }\end{array}$ & $\begin{array}{l}\text { Os resultados indicam que os benefícios } \\
\text { econômicos, o prazer e a sustentabilidade } \\
\text { são preditores da atitude em relação aos } \\
\text { SMC e da intenção comportamental } \\
\text { relativa aos SMC. Notou-se um } \\
\text { relacionamento negativo entre reputação } \\
\text { e atitude em relação aos SMC. Observou-se } \\
\text { uma relação positiva entre atitude quanto } \\
\text { aos SMC e intenção comportamental. }\end{array}$ \\
\hline
\end{tabular}

Fonte: Desenvolvido pelos autores (2019). 


\section{Considerações finais}

A presente pesquisa identificou os fatores motivacionais determinantes para adesão aos SMC no contexto brasileiro. Por meio da condução de uma survey com uma amostra composta de 597 usuários de SMC e utilizando-se da técnica multivariada de AFC, foram obtidos indícios empíricos que possibilitaram validar, no contexto brasileiro, uma escala para mensuração dos determinantes da utilização de SMC. Constatou-se que os benefícios econômicos, a sustentabilidade e a satisfação materialista apresentaram uma influência significativa no que diz respeito à atitude em relação aos SMC e à intenção comportamental dos usuários desses serviços. Foi visto que a reputação, na amostra investigada, não apresentou relação significante com a intenção comportamental e com a atitude dos indivíduos em relação aos SMC.

Portanto, a utilização dos SMC não melhora a imagem do usuário diante da comunidade, assim como também não o faz ganhar respeito, reconhecimento e/ou prestígio. Desse modo, diante dos resultados empíricos obtidos, nota-se que os maiores atrativos para que os usuários estejam propensos a utilizar os SMC estão diretamente relacionados aos ganhos financeiros, às contribuições para a sustentabilidade e à satisfação percebida que esse tipo de serviço pode prover.

Esses resultados mostram-se relevantes, uma vez que apresentam contribuições importantes para a literatura, especialmente para as pesquisas empíricas a respeito da mobilidade compartilhada, tema recente e pouco investigado no contexto brasileiro. A validação da escala no contexto brasileiro assegura um instrumento de pesquisa viável e consistente para futuras replicações, com o intuito compreender, ainda mais, a motivação para utilização dos SMC. No âmbito dos negócios, os resultados deste estudo podem direcionar as empresas para ações de marketing voltadas a uma maior atração de indivíduos que procuram por experiências agradáveis e divertidas, que almejam uma melhor consciência ambiental e que buscam serviços que gerem ganhos econômicos pontuais.

Por fim, como limitações durante a condução do presente estudo, há, nomeadamente, a escolha do tipo de amostra, não probabilística por conveniência com 597 usuários dos SMC, impossibilitando quaisquer iniciativas de realização de generalizações estatísticas dos resultados para além dos participantes do estudo. Portanto, sugere-se a replicação deste estudo em regiões distintas, com amostras mais abrangentes, em determinado tipo de serviço de mobilidade compartilhada ou em outros setores da economia compartilhada.

\section{Referências}

Ajzen, I. (1991). The theory of planned behavior. Organizational Behavior and Human Decision Processes, 50(2), 179-211. http://dx.doi.org/10.1016/0749-5978(91)90020-T.

Bagozzi, R. P., \& Burnkrant, R. E. (1979). Attitude organization and the attitude-behavior relationship. Journal of Personality and Social Psychology, 37(6), 913-929. http://dx.doi.org/10.1037/0022-3514.37.6.913.

Barbosa, C. P. R. (2017). Sharing economy: drivers and barriers to portuguese travelers use peer-to-peer accommodation rentals (Dissertação de mestrado). Instituto Universitário de Lisboa, Lisboa.

Böcker, L., \& Meelen, T. (2017). Sharing for people, planet or profit? Analysing motivations for intended sharing economy participation. Environmental Innovation and Societal Transitions, 23, 28-39.

http://dx.doi.org/10.1016/j.eist.2016.09.004.

Botsman, R., \& Rogers, R. (2011). o que é meu é seu: como o consumo coletivo está mudando o nosso mundo. Porto Alegre: Bookman.

Cadurin, L. D. P. (2016). Demanda potencial para um sistema de compartilhamento de bicicletas pedelecs: o caso de um campus universitário (Dissertação de mestrado). Escola de Engenharia de São Carlos, Universidade de São Paulo, São Carlos. 
Conner, M., Kirk, S. F. L., Cade, J. E., \& Barrett, J. H. (2001). Why do women use dietary supplements? The use of the theory of planned behaviour to explore beliefs about their use. Social Science \& Medicine, 52(4), 621-633. http://dx.doi.org/10.1016/S0277-9536(00)00165-9. PMid:11206658.

Deci, E. L., \& Ryan, R. M. (2000). The" what" and" why" of goal pursuits: human needs and the self-determination of behavior. Psychological Inquiry, 11(4), 227-268. http://dx.doi.org/10.1207/S15327965PLI1104_01.

Demailly, D., \& Novel, A. S. (2014). The sharing economy: make it sustainable. Studies, 3, 14-30.

Engel, F., Blackwell, R. D., \& Miniard, P. W. (2000). Comportamento do consumidor. Rio de Janeiro: LTC.

Fishbein, M. (1963). An investigation of the relationships between beliefs about an object and the attitude toward that object. Human Relations, 16(3), 233-240. http://dx.doi.org/10.1177/001872676301600302.

Fishbein, M. (1967). Attitude and the prediction of behavior. In M. Fishbein (Ed.), Readings in attitude theory and measurement. New York: Wiley.

Fishbein, M., \& Ajzen, I. (1975). Beliefs, attitude, intention and behavior: an introduction to theory and research. Reading: Addison-Wesley.

Fombrun, C. J., \& Rindova, V. P. (2000). The road to transparency: reputation management at Royal Dutch/Shell. The Expressive Organization, 7, 7-96.

Fornell, C., \& Larcker, D. F. (1981). Evaluating structural equation models with unobservable variables and measurement error. Journal of Marketing Research, 18(1), 39-50.

http://dx.doi.org/10.1177/002224378101800104.

Freese, C., Schönberg, A. T., \& Horstkötter, D. (2014). Shared mobility: how new businesses are rewriting the rules of the private transportation game. München: Roland Berger Strategy Consultants.

Freitas, C. S., Petrini, M. C., \& Silveira, L. M. (2016). Desvendando o consumo colaborativo: Uma proposta de tipologia. In Anais do 9o Congresso Latino-Americano no Varejo. São Paulo: FGV-EAESP.

Hair, J. F., Jr., Hult, G. T. M., Ringle, C. M., \& Sarstedt, M. (2016). A primer on partial least squares structural equation modeling (PLS-SEM). Thousand Oaks: Sage Publications.

Hamari, J., Sjöklint, M., \& Ukkonen, A. (2016). The sharing economy: why people participate in collaborative consumption. Journal of the Association for Information Science and Technology, 67(9), 2047-2059.

http://dx.doi.org/10.1002/asi.23552.

Heinrichs, H. (2013). Sharing economy: a potential new pathway to sustainability. GAIA-Ecological Perspectives for Science and Society, 22(4), 228-231. http://dx.doi.org/10.14512/gaia.22.4.5.

Hultman, M., Kazeminia, A., \& Ghasemi, V. (2015). Intention to visit and willingness to pay premium for ecotourism: the impact of attitude, materialism, and motivation. Journal of Business Research, 68(9), 1854-1861. http://dx.doi.org/10.1016/j.jbusres.2015.01.013.

Kim, J., Yoon, Y., \& Zo, H. (2015). Why people participate in the sharing economy: a social exchange perspective. In Proceedings of the Pacific Asia Conference on Information Systems. PACIS.

Le Vine, S., \& Polak, J. (2015). Introduction to special issue: new directions in shared480 mobility research. Transportation, 42(3), 407-411. http://dx.doi.org/10.1007/s11116-015-9603-4.

Liang, Z., \& Shi, W. (2005). PET: a personalized trust model with reputation and risk evaluation for P2P resource sharing. In Proceedings of the 38th Annual Hawaii International Conference on System Sciences (HICSS'05) (pp. 201b-201b). New York: IEEE.

Lopes, M. S., \& Alves, C. A. (2017). Das redes sociais ao Instagram, da intenção ao comportamento: um estudo sobre a influência do eWOM (boca-a-boca eletrônico) na intenção de visitar um restaurante. In Anais do XX Seminários em Administração (Semead) (Vol. 1, pp. 1-20). São Paulo: FEA-USP.

Malhotra, N. K. (2012). Pesquisa de marketing: uma orientação aplicada. (6. ed.). Porto Alegre: Bookman. 
Mann, F. C. F. (2018). Economia compartilhada e consumo colaborativo no Brasil: análise das motivações e características dos seus participantes (Dissertação de mestrado). Universidade Federal Fluminense, Niterói.

Maroco, J. (2010). Análise estatística: com utilização do SPSS. Lisboa: Edições Silabo.

Maurer, A. M., Figueiró, P. S., Campos, S. A. P., Silva, V. S., \& Barcellos, M. D. (2015). Yes, we also can! 0 desenvolvimento de iniciativas de consumo colaborativo no Brasil. BASE: Revista de Administração e Contabilidade da UNISINOS, 12(1), 68-80.

Mello, E. S. (2008). Mobilidade urbana sustentável em projetos estruturantes: analise urbanística e ambiental do corredor de transporte da Avenida Bernardo Vieira - Natal/RN (Dissertação de mestrado). Universidade Federal do Rio Grande do Norte, Natal.

Miranda, M. A. R. (2018). Fatores que influenciam a atitude no Consumo Colaborativo no Brasil (Dissertação de mestrado). Fundação Instituto Capixaba de Pesquisas em Contabilidade, Economia e Finanças, Vitória.

Moeller, S., \& Wittkowski, K. (2010). The burdens of ownership: reasons for preferring renting. Managing Service Quality, 20(2), 176-191. http://dx.doi.org/10.1108/09604521011027598.

Mohlmann, M. (2015). Collaborative consumption: determinants of satisfaction and the likelihood of using a sharing economy option again. Journal of Consumer Behaviour, 14(3), 193-207. http://dx.doi.org/10.1002/cb.1512.

Moura, L. R. (2017). Mobilidade Urbana e Política Pública: uma análise de bicicletas integradas na perspectiva da sustentabilidade. In Anais da VIII Jornada Internacional de Políticas Públicas. São Luis: UFMA.

Murphy, C. (2016). Shared mobility and the transformation of public transit. Washington: National Academy of Sciences. http://dx.doi.org/10.17226/23578.

Pinotti, R. C. (2016). Hospitalidade e a intenção de recompra no contexto da economia compartilhada: um estudo em meios de hospedagem alternativos com equações estruturais (Dissertação de mestrado). Universidade Anhembi Morumbi, São Paulo.

Pizzol, H. O. D. (2015). Proposição de uma escala para mensuração do consumo colaborativo: compreendendo o compartilhamento de bens e a sua relação com os valores pessoais (Dissertação de mestrado). Faculdade de Administração, Contabilidade e Economia, Pontifícia Universidade Católica do Rio Grande do Sul, Porto Alegre.

Ribas, J. R., \& Vieira, P. R. C. (2011). Análise multivariada com o uso do SPSS. Rio de Janeiro: Editora Ciência Moderna.

Ringle, C., Silva, D., \& Bido, D. (2014). Modelagem de equações estruturais com utilização do Smartpls. Revista Brasileira de Marketing, 13(2), 54-71.

Sampaio, L. C., Fischer, W., \& Miura, M. N. (2018). Comportamento do consumidor na economia compartilhada: por que as pessoas participam? In Anais do XXIX Encontro Nacional dos Cursos de Graduação em Administração (ENANGRAD). São Paulo: ANGRAD.

Shaheen, S., Cohen, A., \& Zohdy, I. (2016). Shared mobility: current practices and guiding principles. Washington: U.S. Department of Transportation.

So, K. K. F., Oh, H., \& Min, S. (2018). Motivations and constraints of Airbnb consumers: findings from a mixedmethods approach. Tourism Management, 67, 224-236. http://dx.doi.org/10.1016/j.tourman.2018.01.009.

Tussyadiah, I. P., \& Pesonen, J. (2018). Drivers and barriers of peer-to-peer accommodation stay-an exploratory study with American and Finnish travellers. Current Issues in Tourism, 21(6), 703-720.

http://dx.doi.org/10.1080/13683500.2016.1141180.

Wang, W. H., \& Liu, Y. J. (2009). Attitude, behavioral intention and usage: an empirical study of Taiwan Railway's internet ticketing system (pp. 72). Taiwan: National Taiwan Ocean University.

Wang, X., \& Yang, Z. (2008). Does country-of-origin matter in the relationship between brand personality and purchase intention in emerging economies? Evidence from China's auto industry. International Marketing Review, 25(24), 458-474. http://dx.doi.org/10.1108/02651330810887495. 
Yakin, V., Kacar, A. İ., \& Ay, C. (2017). Sharing Economy: why the Turkish consumers use Airbnb? Journal of Marketing and Consumer Behaviour in Emerging Markets, 6(2), 25-36. http://dx.doi.org/10.7172/24496634.jmcbem.2017.2.2.

Yang, S., \& Ahn, S. (2016). Impact of motivation in the sharing economy and perceived security in attitude and loyalty toward Airbnb. Advanced Science and Technology Letters, 129, 180-184.

http://dx.doi.org/10.14257/astl.2016.129.36.

Editor: Fábio Duarte

Recebido: Fev. 12, 2019

Aprovado: Maio 29, 2019 